\title{
Drug resistance in community-acquired respiratory tract infections: role for an emerging antibacterial
}

This article was published in the following Dove Press journal:

Infection and Drug Resistance

17 June 2010

Number of times this article has been viewed

\author{
Lorenzo Aguilar' \\ María-José Giménez' \\ José Barberán² \\ 'Microbiology Department, School of \\ Medicine, University Complutense, \\ Madrid; ' 2 nfectious Diseases \\ Department, Hospital Central de la \\ Defensa Gomez Ulla, Madrid, Spain
}

Correspondence: Lorenzo Aguilar Microbiology Department, School of Medicine, University. Complutense, Avda. Complutense s/n, 28040 Madrid, Spain

Tel +34 913 94I 505

Fax +34 913 94I 5II

Email laguilar@med.ucm.es

\begin{abstract}
The nasopharynx is the ecological niche where evolution towards resistance occurs in respiratory tract isolates. Dynamics of different bacterial populations in antibiotic-free multibacterial niches are the baseline that antibiotic treatments can alter by shifting the competitive balance in favor of resistant populations. For this reason, antibiotic resistance is increasingly being considered to be an ecological problem. Traditionally, resistance has implied the need for development of new antibiotics for which basic efficacy and safety data are required prior to licensing. Antibiotic development is mainly focused on demonstrating clinical efficacy and setting susceptibility breakpoints for efficacy prediction. However, additional information on pharmacodynamic data predicting absence of selection of resistance and of resistant subpopulations, and specific surveillance on resistance to core antibiotics (to detect emerging resistances and its link with antibiotic consumption in the community) are valuable data in defining the role of a new antibiotic, not only from the perspective of its therapeutic potential but also from the ecologic perspective (countering resistances to core antibiotics in the community). The documented information on cefditoren gleaned from published studies in recent years is an example of the role for an emerging oral antibacterial facing current antibiotic resistance in community-acquired respiratory tract infections.
\end{abstract}

Keywords: respiratory tract infection, antibiotic resistance, cefditoren, community

\section{Nasopharyngeal colonization and prevalent isolates}

Mucosal surfaces are simultaneously colonized by multiple species, with an intricate balance in the nasopharynx between Streptococcus pneumoniae, Streptococcus pyogenes, Haemophilus influenzae, and other nasopharyngeal flora. These bacterial species share three characteristics, ie, they colonize the nasopharynx of humans as their exclusive host, are exogenously transmitted to colonize the nasopharynx of new hosts, and are common etiological agents (most prevalent isolates) of bacterial community-acquired respiratory tract infections when they endogenously migrate to different ecosystems or when changes within their natural ecosystem occur. Thus the upper respiratory tract, with its commensal flora, acts both as a colonization defense mechanism and as a primary bacterial source for respiratory tract infections, S. pyogenes being the etiologic agent of tonsillitis, and $S$. pneumoniae and $H$. influenzae being the causative agents of otitis media, sinusitis, and lower respiratory tract infections, ie, acute exacerbation of chronic bronchitis (AECB) and pneumonia.

Carriage of these common respiratory isolates depends on multiple factors, such as active or passive smoking, crowding, age, bacterial fitness, specific vaccination, and 
bacterial interference in antibiotic-free niches. ${ }^{1-3}$ Approximately $80 \%$ of healthy individuals carry $H$. influenzae, ${ }^{4}$ with multiple strains in 50\% positive samples and a high turnover of strains. ${ }^{2,5}$ In the case of $S$. pneumoniae, carriage ranges from $10 \%$ to $40 \%$ in an age-dependent manner, ${ }^{6}$ with lower percentages of multiple strains in the same sample ${ }^{7}$ and a duration of nasopharyngeal carriage depending on age, seasonality, resistance to penicillin, serotype, and simultaneous carriage by other family members. ${ }^{8,9}$ S. pyogenes frequently colonizes the nasopharynx of asymptomatic persons, with carriage rates of $15 \%-20 \%$ in school children (crowding favors interpersonal spread), but this is considerably lower in adults. ${ }^{10}$

\section{Bacterial evolution towards resistance in respiratory tract isolates}

The nasopharynx is the ecological niche where evolution towards resistance occurs in respiratory tract isolates. The evolution of antibiotic resistance involves two processes, ie, emergence and spread. ${ }^{11,12}$ Resistance to $\beta$-lactams and macrolides is mostly due to acquisition of exogenous resistance genes, and has been described in S. pneumoniae and $H$. influenzae (in both cases, resistance to both $\beta$-lactams and to macrolides) and in S. pyogenes (resistance to macrolides), ${ }^{13-16}$ with de novo resistance occurring rarely within a given host in a susceptible bacterial population. ${ }^{13}$ In contrast, resistance to fluoroquinolones in S. pneumoniae arises within a given host due to point mutations. ${ }^{13}$

Evolution is based on production of variation, management of variation, and natural selection of variants. ${ }^{17}$ Dynamics of different bacterial populations in antibiotic-free niches are the baseline that antibiotic treatments can alter ${ }^{18,19}$ by shifting the competitive balance in favor of resistant populations. For this reason, antibiotic resistance is increasingly regarded as an ecologic problem. Once resistance has emerged, physiologic concentrations of different antibiotics may select resistant strains by eradicating the susceptible ones (thus unmasking resistant populations), ${ }^{19}$ or by selecting intrastrain-resistant subpopulations. ${ }^{20}$ Because multiple individuals harbor multiple bacterial populations exhibiting different degrees of antibiotic resistance at the community level, the prevalence of resistance is directly related to antibiotic consumption in the community. ${ }^{21}$

\section{Antibiotic consumption as the driver of resistance: a global problem}

Infection, mainly of the respiratory tract, ${ }^{22,23}$ is the most frequent reason for seeking medical attendance in the community.
Around $85 \%-90 \%$ of antibiotic consumption occurs in the community, and $80 \%$ of this consumption is for the treatment of respiratory tract infections. ${ }^{24}$ Antibiotic consumption has been associated with resistance selection. ${ }^{21}$ Consumption of $\beta$-lactams and macrolides has been associated with penicillin/ erythromycin resistance in S. pneumoniae, both temporally ${ }^{25}$ and geographically, ${ }^{26}$ with high correlations between penicillin/erythromycin resistance and consumption of long half-life macrolides and second-generation oral cephalosporins. ${ }^{25,26}$ Associations between ampicillin/amoxicillin resistance in H. influenzae and Moraxella catarrhalis and consumption of aminopenicillins (with or without clavulanic acid), ${ }^{27,28}$ as well as the association between erythromycin resistance in $S$. pyogenes and consumption of long half-life macrolides, have also been described. ${ }^{29,30}$

Because antibiotic consumption in the community has been associated with resistance prevalence, in geographic locations with high antibiotic consumption, eg, Spain, associations between the resistance rates found in different bacterial species of respiratory tract isolates could be expected. In Spain, penicillin resistance in S. pneumoniae has been significantly associated with erythromycin resistance (due to the coresistance selection phenomenon), ${ }^{31,32}$ and with ampicillin resistance in $H$. influenzae (due to the phenomenon of coselection of resistance). In addition, geographically, erythromycin resistance in $S$. pyogenes was significantly related to penicillin and/or erythromycin resistance in S. pneumoniae and to ampicillin resistance in H. influenzae. ${ }^{13}$ Considering these associations, in Spain, as in other countries, resistance should be considered as a global problem with regard to respiratory isolates in the community.

\section{Current resistances in respiratory isolates in the community}

\section{Streptococcus pneumoniae}

Penicillin/erythromycin nonsusceptibility in $S$. pneumoniae is mainly clustered in a reduced number of serotypes. In invasive isolates, the increase in the prevalence of antibiotic nonsusceptibility and of certain serotypes that occurred in the 1980s and 1990s related to antibiotic consumption reversed in the 2000s when the seven-valent conjugate pneumococcal vaccine (PCV7) (including serotypes most associated with penicillin/erythromycin nonsusceptibility) was introduced for childhood immunization. ${ }^{33,34}$ The introduction of PCV7 produced not only a dramatic reduction in the incidence of invasive pneumococcal disease, but also a marked decrease in PCV7 serotypes which consequently affected penicillin and erythromycin nonsusceptibility in invasive isolates. ${ }^{34,35}$ 
With respect to noninvasive isolates in Spain, surveillance including high number of isolates has shown that nonsusceptibility to penicillin was $45 \%-50 \%$ from 1998 to 2002 , with full oral penicillin resistance rates of approximately $20 \% .^{31,36}$ Amoxicillin nonsusceptibility remained around $10 \%$ in this period but, among full penicillin-resistant isolates, this rate increased to around $40 \%{ }^{31,36}$ These penicillin-resistant isolates exhibited nearly $100 \%$ resistance to oral secondgeneration cephalosporins, eg, cefaclor or cefuroxime, and $55 \%$ resistance to macrolides. ${ }^{31}$ In a worldwide surveillance (1999-2004) of isolates from community-acquired infections in patients $\geq 65$ years (including a high number of noninvasive isolates), penicillin nonsusceptibility was approximately $22 \%$ in Eastern Europe and North America, and up to about $60 \%$ in Far East. ${ }^{37}$

The emergence of amoxicillin resistance within pre-existing penicillin-resistant clones has also been related to macrolide and ciprofloxacin resistance,,$^{31,36,38}$ with reports of spread of troublesome clones with MIC values of amoxicillin higher than those of penicillin, ${ }^{39}$ mainly the four Spanish multiresistant ones $\left(\right.$ Spain $^{23 \mathrm{~F}}-1$, Spain ${ }^{6 \mathrm{~B}}-2$, Spain ${ }^{9 \mathrm{~V}}-3$, and Spain $^{14}-5$ ).

Erythromycin resistance in pneumococci has remained relatively stable in Spain, with rates of around 35\% between 1996 and 2002 in surveillances including high numbers of noninvasive isolates..$^{31,36,40}$ The main resistant phenotype is $\mathrm{MLS}_{\mathrm{B}}$ (approximately 90\%), with M-efflux representing approximately $10 \%,{ }^{31}$ thus erythromycin resistance also implies clarithromycin and azithromycin resistance. Similar resistance rates are found in the US (30.0\%-35.3\%), with an increase in highly resistant strains in recent years, and a decrease in M-efflux-mediated resistant strains. ${ }^{41}$

In the same surveillance, ciprofloxacin resistance (MIC $\geq 4 \mu \mathrm{g} / \mathrm{mL}$ ) was around $5 \%-7 \%,{ }^{31,37}$ but $>85 \%$ of these isolates were susceptible to levofloxacin and moxifloxacin. From these data it is deduced that rates of nonsusceptibility to respiratory fluoroquinolones (levofloxacin and moxifloxacin) are not $>1 \% .{ }^{36}$ Worldwide, a $2.2 \%$ nonsusceptibility rate has been reported for levofloxacin. ${ }^{37}$

Multiple resistance, defined as full resistance to two or more of the six classes of antibacterials represented by penicillin, erythromycin, cefuroxime, tetracyclines, trimethoprimsulfamethoxazole, and levofloxacin, has been reported in S. pneumoniae, with rates as high as $19.1 \%$ in North America, $27.7 \%$ in Western Europe, and $80.4 \%$ in the Far East. ${ }^{37}$

\section{Streptococcus pyogenes}

S. pyogenes is highly susceptible to all $\beta$-lactams. Prevalence of resistance to erythromycin was $37 \%$ in $2001-2002$ in Spain, ${ }^{31}$ with the M-efflux phenotype being the most prevalent (86\%), and with $14 \%$ of strains from the MLS $_{B}$ phenotype showing constitutive resistance. ${ }^{31}$ Since both mechanisms imply resistance to 14- and 15-membered macrolides, erythromycin resistance implies resistance to azithromycin and clarithromycin. ${ }^{42}$

In the last few years, $S$. pyogenes isolates showing lowlevel resistance to fluoroquinolones have been reported, ${ }^{43-45}$ with infrequent high-level resistance to date. However, due to the high prevalence of isolates harboring $\operatorname{par} \mathrm{C}$ mutation, in the near future the frequency of high-level resistance may increase because only one new mutation in gyrA is required ${ }^{46}$ In Spain, there has been a marked increase in the number of isolates with low-level resistance, and isolates showing high-level resistance have also been detected. ${ }^{47}$

\section{Haemophilus influenzae}

The basic problem of resistance in this species is defined by ampicillin as the resistance marker. According to successive surveillance studies carried out from 1996 to 2002, approximately $25 \%$ of $\mathrm{H}$. influenzae isolates are resistant to ampicillin in Spain..$^{31,48,49}$ Up to $80 \%$ of these ampicillinresistant isolates produce $\beta$-lactamases (TEM-1, TEM-2, and with lower frequency ROB-1) that are inhibited by clavulanic acid. The remaining $20 \%$ ampicillin-resistant isolates $(5 \%$ of all $H$. influenzae isolates in Spain) are resistant to ampicillin due to mutations in the ftsI gene that cause alterations in the amino acid sequences of penicillin-binding protein 3 (PBP3). ${ }^{50}$ This resistance genotype defines BLNAR ( $\beta$-lactamase negative ampicillin-resistant) strains and since alterations in PBP3 preclude the adequate binding of ampicillin and amoxicillin, BLNAR strains are also resistant to amoxicillin-clavulanic acid, ampicillin-sulbactam, cefaclor, and cefuroxime. ${ }^{51}$

Both mechanisms of resistance, ie, $\beta$-lactamase production and mutations in the ftsI gene, are present in BLPACR ( $\beta$-lactamase positive amoxicillin-clavulanic acid resistant) strains. Among Spanish isolates, according to data from different studies, there is an increasing prevalence of BLNAR (from 10\% in 1997-1998 to approximately 30\% in 2004-2005) and to a lesser extent BLPACR phenotypes, ${ }^{52,53}$ in relation to $\beta$-lactam consumption over time, mainly amoxicillin with or without clavulanic acid..$^{53}$

\section{Efficacy prediction for commonly used antibiotics}

While "microbiologic breakpoints" detect wild-type bacterial populations that do not harbor any acquired or selected resistance to the antibacterial examined, 
"pharmacokinetic/pharmacodynamic (PK/PD) breakpoints" have been associated with microbial killing as an endpoint to predict bacterial eradication and clinical outcome. ${ }^{54-56}$ By relating pharmacokinetic variables and susceptibility data (ie, antibiotic drug exposure relative to in vitro MIC), $\mathrm{PK} / \mathrm{PD}$ breakpoints indicate the highest MIC value that produces the adequate value for the relevant $\mathrm{PK} / \mathrm{PD}$ parameter. In the case of $S$. pneumoniae and $H$. influenzae, and $\beta$-lactams or macrolides, the time (expressed as the percentage of the dosing interval) that antibiotic concentrations exceed the value of MIC ( $>$ MIC) is the parameter predicting efficacy, with a cutoff value of $40 \%$, but in the case of fluoroquinolones, the parameter is the relationship between the area under the serum concentration-time curve (AUC) and the MIC (AUC/MIC), with a cutoff value of 30 .

In a multicenter surveillance study in Spain, similar susceptibility rates were found for $S$. pneumoniae by applying the breakpoints defined by the Clinical Laboratory Standards Institute (CLSI) and PK/PD breakpoints for amoxicillin (approximately 92\%), cefuroxime-axetil (about 67\%), erythromycin and azithromycin (about 64\%), but not for cefaclor (61.7\% versus $40.5 \%){ }^{31}$

For H. influenzae, the use of CLSI or PK/PD breakpoints does not influence susceptibility rates of ciprofloxacin (100\%), ampicillin (about 75\%) or amoxicillin-clavulanic acid (about 97\%), but changes the rates of susceptibility to cefuroxime-axetil (from $100 \%$ to $72.8 \%$ ) and cefaclor (from $82.1 \%$ to $1.4 \%$ ), with lower rates when applying PK/PD breakpoints. ${ }^{31}$ In the case of macrolides, differences are clearly evident, with reductions in susceptibility rates to clarithromycin and azithromycin from 72\% 100\%, respectively, by applying CLSI breakpoints, to $2.2 \%$ and $1.2 \%$, respectively, by applying $\mathrm{PK} / \mathrm{PD}$ breakpoints. ${ }^{31}$

\section{Management strategies to overcome resistance}

Under circumstances of a global problem of resistance among prevalent isolates in community respiratory pathogens, there is a need for strategies countering resistance, ie, selection of coresistance within the same species and coselection of resistances between species. One possible strategy is based on increasing oral doses for the treatment of respiratory tract infections in the community that, although not adequate for macrolides (high-level resistance), has been used in the case of amoxicillin-clavulanic acid, with the development of new formulations adequate to minimize amoxicillin resistance in $S$. pneumoniae, but inadequate to counter BLNAR and BLPACR diffusion among $H$. influenzae. Another possible strategy is the development of new antibiotics with adequate pharmacokinetics and high in vitro activity against community prevalent isolates, achieving values of pharmacodynamic parameters predicting bacterial eradication. The need for new antibiotics in the community is mainly defined by antimicrobial activity against the prevalent resistance phenotypes rather than the activity against phenotypes susceptible to antibiotics previously used in the community. For this reason new antibiotics for the treatment of respiratory tract infections should demonstrate in vitro activity against $S$. pneumoniae not susceptible to previous antibiotics (with specific phenotypes of resistance and clones) and $H$. influenzae nonsusceptible to ampicillin (BLNAR, BLPACR).

\section{Cefditoren for community-acquired respiratory tract infections}

\section{In vitro activity}

Cefditoren is an oral, third-generation aminothiazolyl cephalosporin with structural components similar to those of first- and third-generation cephalosporins. ${ }^{57}$ In general, cephalosporins differ from one another mainly in the two side chain components attached to the cephem scaffold. In cefditoren, the group attached at the $\mathrm{C}-7$ position affords activity against Gram-negative microorganisms, whereas the one attached at the $\mathrm{C}-3$ position affords activity against Gram-positive bacteria (not seen in other non-first-generation cephalosporins).

In vitro studies carried out to explore the activity of cefditoren included not only a high number of strains isolated in the community but also a significant number of strains with troublesome resistance phenotypes/genotypes. Cefditoren exhibited potent intrinsic activity, inhibiting all penicillin-susceptible $S$. pneumoniae at concentrations of $0.12 \mu \mathrm{g} / \mathrm{mL}\left(\mathrm{MIC}_{90}\right.$ of $\left.\leq 0.03 \mu \mathrm{g} / \mathrm{mL}\right) .{ }^{58}$ At concentrations of $0.5 \mu \mathrm{g} / \mathrm{mL}$, cefditoren inhibited $92.6 \%$ of cefotaxime nonsusceptible pneumococci and $>97 \%$ of strains nonsusceptible to the other antibiotics (penicillins, cephalosporins, macrolides, ketolides, and quinolones). ${ }^{59}$ With respect to Spanish multiresistant clones, cefditoren exhibited an $\mathrm{MIC}_{90}$ of $\leq 0.5 \mu \mathrm{g} / \mathrm{mL}$ against strains belonging to Spain $^{23 \mathrm{~F}}$-1 (with susceptibility rates to amoxicillin-clavulanic acid of $4.2 \%$, to macrolides of $66.7 \%$, and to cefotaxime of $95.8 \%$ ), Spain ${ }^{9 \mathrm{~V}}-3$ (with susceptibility rates to amoxicillin-clavulanic acid of $30 \%$, to macrolides of $81.7 \%$, and to cefotaxime of $85 \%$ ), and Spain $^{6 \mathrm{~B}}-2$ (with susceptibility rates to amoxicillin-clavulanic acid of $6.8 \%$, to macrolides of $4.5 \%$, and to cefotaxime of $81.8 \%) .{ }^{60}$ Against the most troublesome strains of the clone Spain ${ }^{14}-5$ (that exhibited susceptibility rates of $7.3 \%$ 
to amoxicillin, $4.9 \%$ to macrolides, $57.3 \%$ to cefotaxime, and only $65.9 \%$ to levofloxacin), cefditoren $\mathrm{MIC}_{50} / \mathrm{MIC}_{90}$ values were $0.5 / 1 \mu \mathrm{g} / \mathrm{mL}$, one dilution lower than values for cefotaxime. $^{60}$

Against $H$. influenzae, while amoxicillin-clavulanic acid and cefuroxime $\mathrm{MIC}_{50} / \mathrm{MIC}_{90}$ values increased from $0.5 / 1 \mu \mathrm{g} / \mathrm{mL}$ for ampicillin-susceptible strains to $2 / 4$ and $1 / 4 \mu \mathrm{g} / \mathrm{mL}$, respectively, for BLNAR strains, and up to $4 / 8$ and $4 / 16 \mu \mathrm{g} / \mathrm{mL}$, respectively, for BLPACR strains, cefditoren exhibited similar intrinsic activity to that of cefotaxime against ampicillin-susceptible, BLNAR and BLPACR strains with $\mathrm{MIC}_{50} / \mathrm{MIC}_{90}$ values of $0.03 / 0.06 \mu \mathrm{g} / \mathrm{mL} .^{61,62}$ The excellent intrinsic activity of cefditoren against $H$. influenzae has recently been confirmed in a multicenter European study testing 665 clinical isolates, with $\mathrm{MIC}_{50} / \mathrm{MIC}_{90}$ for cefditoren of $\leq 0.06 / \leq 0.06 \mu \mathrm{g} / \mathrm{mL}^{63}$

\section{Pharmacokinetics and pharmacodynamics}

In a Phase I study administering a single dose of $400 \mathrm{mg}$ cefditoren-pivoxil with food to 10 healthy Caucasian male volunteers, Cmax was $3.7 \pm 0.7 \mu \mathrm{g} / \mathrm{mL}, \mathrm{T}_{\max }$ was 2 hours, $\mathrm{AUC}_{0-\alpha}$ was $12.5 \pm 1.6 \mu \mathrm{g} \times$ hour $/ \mathrm{mL}$, and the elimination half-life was $1.54 \pm 0.20$ hours. ${ }^{64}$ In the theoretical pharmacodynamic assessment performed with these data, considering $\mathrm{T}>\mathrm{MIC}$ as the relevant $\mathrm{PK} / \mathrm{PD}$ parameter, the $400 \mathrm{mg}$ bid regimen of cefditoren-pivoxil obtained a value of T $>$ MIC for total drug of approximately $55 \%$ for MIC $0.5 \mu \mathrm{g} / \mathrm{mL}$, $68 \%$ for MIC $0.25 \mu \mathrm{g} / \mathrm{mL}, 81 \%$ for MIC $0.12 \mu \mathrm{g} / \mathrm{mL}$ and $94 \%$ for MIC $0.06 \mu \mathrm{g} / \mathrm{mL}^{.64}$

This pharmacodynamic analysis was performed considering the total drug. Cefditoren is a highly protein-bound antimicrobial with $88 \%$ protein binding. ${ }^{57}$ It has been suggested that only the unbound fraction of an antimicrobial is active in vitro, but the reversibility of protein binding implies that limitation of activity may be far from absolute, even in highly protein-bound agents. ${ }^{65}$ To explore the activity of cefditoren in the presence of human albumin, a one-compartmental in vitro dynamic model simulating the cefditoren $400 \mathrm{mg}$ bid serum profile over 24 hours, using media consisting of $75 \%$ human serum and $25 \%$ broth with albumin at physiologic concentration (4.9 g/dL), was used. ${ }^{66}$ Antibacterial activity was determined over time against $S$. pneumoniae exhibiting MICs of 0.25 and $0.5 \mu \mathrm{g} / \mathrm{mL} .{ }^{66}$ The cefditoren protein binding in the system was $87.1 \%$, thus potentially interfering with cefditoren activity as in in vivo situations. Under these circumstances, at 24 hours, initial inoculum reductions for strains with MIC of $0.25 \mu \mathrm{g} / \mathrm{mL}$ was $>99.9 \%$ (bactericidal activity), and ranged from 53\% to 97\% (an effect higher than simply bacteriostatic) for strains with MIC $0.5 \mu \mathrm{g} / \mathrm{mL}{ }^{66}$

For extrapolation to humans, a Monte Carlo simulation, ie, the method for determining the probability to achieve a specific value of a PK/PD index in the general population, was performed using cefditoren data from a Phase I study, ${ }^{64}$ and considering both total and free (calculated using the rate of protein binding) concentrations of cefditoren. ${ }^{67}$ Considering the target attainment of $\mathrm{T}>\mathrm{MIC} \geq 40 \%$ (as predictive of efficacy), ${ }^{68,69}$ cefditoren covered ( $>90 \%$ probability to achieve this value of T $>$ MIC) strains with MIC values of $\leq 0.5 \mu \mathrm{g} / \mathrm{mL}$ (total drug), and $\leq 0.12 \mu \mathrm{g} / \mathrm{mL}$ (free drug). When the bacteriostatic target attainment $(33 \% \mathrm{~T}>\mathrm{MIC})^{70}$ was considered, based on definitions of "susceptibility" by the $\mathrm{FDA}^{71}$ and $\mathrm{CLSI}^{51}$ as "pathogen likely inhibition by blood concentrations", cefditoren had a $>90 \%$ probability to achieve this bacteriostatic endpoint for MICs $\leq 0.5 \mu \mathrm{g} / \mathrm{mL}$ and $\leq 0.25 \mu \mathrm{g} / \mathrm{mL}$ for total and free drug, respectively. ${ }^{67}$

\section{Cefditoren breakpoints}

Experimental data (in vitro susceptibility and PK/PD experimental data) and Monte Carlo extrapolations are valuable data for assessing potential breakpoints for cefditoren. Different values have been proposed or defined for cefditoren. While breakpoint values proposed by the FDA are $\leq 0.12 \mu \mathrm{g} / \mathrm{mL}$ for susceptibility and $\geq 0.5 \mu \mathrm{g} / \mathrm{mL}$ for resistance, some authors have suggested cefditoren susceptibility breakpoint values of $\leq 0.5 \mu \mathrm{g} / \mathrm{mL}$ or $\leq 1 \mu \mathrm{g} / \mathrm{mL},{ }^{72-74}$ considering cefditoren $\mathrm{MIC}_{90}$ values lower than the breakpoint values for parenteral third-generation cephalosporins and the pharmacokinetics of cefditoren. Although nowadays there are no established breakpoints defined by the CLSI or the European Committee on Antimicrobial Susceptibility Testing, experimental and Monte Carlo results are in accordance with the susceptibility breakpoint approved by the Spanish Agency during the registration procedure in Europe (susceptibility $\leq 0.5 \mu \mathrm{g} / \mathrm{mL}$ ). ${ }^{75}$ With this breakpoint value, $100 \%$ isolates of $H$. influenzae and S. pyogenes and $94 \%$ of S. pneumoniae are covered in Spain. ${ }^{76}$

\section{Clinical data on cefditoren in community-acquired respiratory tract infections Upper respiratory tract infections}

Data from all six clinical trials carried out during the clinical development of cefditoren in upper respiratory tract infections were combined in a pooled analysis. ${ }^{77}$ With 
respect to pharyngotonsillitis, no significant differences in clinical response were found between cefditoren and penicillin $\mathrm{V}$, with success rates ranging from $89.4 \%$ to $95.3 \%$ when pooling data from the three comparative multicenter studies (two previously published) ${ }^{78,79}$ already performed. ${ }^{77}$ Eradication of $S$. pyogenes was higher with cefditoren at the end of therapy $(90.4 \%$ versus $82.7 \% ; P=0.002)$ and at the end of follow-up ( $84.7 \%$ versus $76.7 \% ; P=0.008)$, although statistical significance (set at $P<0.001$ ) was not reached. ${ }^{77}$

Similarly, in acute sinusitis, no differences in clinical response were found between cefditoren and comparators (cefuroxime or amoxicillin-clavulanic acid) both at the end of therapy ( $80.2 \%$ versus $84.8 \%$ ) and at the end of follow-up ( $71.2 \%$ versus $77.4 \%$ ) pooling data from the three studies (one previously published) ${ }^{80}$ performed during the clinical development of cefditoren. ${ }^{77}$

\section{Lower respiratory tract infections}

Seven studies were carried out in the clinical development of cefditoren for the treatment of lower respiratory tract infections, four studies in community-acquired pneumonia (CAP), and three studies in AECB. A pooled analysis of data was performed including a total of 4159 randomized patients. ${ }^{81}$ In CAP studies (two previously published), ${ }^{82,83}$ no significant differences were found in pooled clinical response rates between cefditoren and comparators (amoxicillin-clavulanic acid or cefpodoxime), with percentages of responders ranging from $89.2 \%$ to $91.8 \%$ at the end of therapy, and from $85.9 \%$ to $90.4 \%$ at the end of follow-up.

In AECB, pooled data from the three published studies ${ }^{84-86}$ showed clinical response rates ranging from $85.8 \%$ to $91.3 \%$ at the end of therapy, and from $81.2 \%$ to $83.3 \%$ at the end of follow-up, without significant differences between cefditoren and the comparators, ie, cefuroxime or clarithromycin.

CAP and AECB data were pooled to explore microbiologic outcomes. ${ }^{81}$ With respect to $S$. pneumoniae, there were no significant between-group differences in the rate of bacteriologic responders, with rates ranging from $88.5 \%$ to 92.0\%. All penicillin nonsusceptible (MIC $\geq 0.12 \mu \mathrm{g} / \mathrm{mL}$ ) isolates of $S$. pneumoniae in the cefditoren $400 \mathrm{mg}$ group $(\mathrm{n}=20), 16$ of 19 strains $(84.2 \%)$ in the cefditoren $200 \mathrm{mg}$ group, and 16 of 17 strains $(94.1 \%$ ) in the comparator group were eradicated or presumed to be eradicated. ${ }^{81}$ Among penicillin-resistant ( $\mathrm{MIC} \geq 2 \mu \mathrm{g} / \mathrm{mL}$ ) isolates, 17 of 18 $(94.4 \%)$ isolates in both cefditoren arms were eradicated or presumed to be eradicated compared with 10 of $11(90.9 \%)$ in the comparator group. ${ }^{81}$
No significant differences in microbiologic outcome with respect to $H$. influenzae were found between groups, with pooled response rates ranging from $82.7 \%$ to $86.6 \%{ }^{81}$

\section{Safety profile}

Safety data from all the 13 clinical trials carried out with cefditoren in the treatment of community-acquired respiratory tract infections were analyzed in a pooled analysis. ${ }^{87}$ The safety population was defined as all randomized patients with at least one dose intake, and consisted of 4592 patients for cefditoren. Cefditoren exhibited an adverse event profile similar to that of other antibiotics currently used in the treatment of community-acquired respiratory tract infections, with diarrhea being the most frequent adverse event (9.9\%) followed by nausea (3.5\%), abdominal pain (1.8\%), and dyspepsia $(1.1 \%) .{ }^{87}$ The rate of vaginosis reported in the female population was $3.9 \% .{ }^{87}$

\section{Conclusions}

There is increasingly evidence confirming that bacterial eradication should be the primary goal of antibiotic therapy because eradication is the main determinant of both therapeutic outcome and prevention of resistance. In the most prevalent bacterial isolates from communityacquired respiratory tract infections, that are responsible for $80 \%$ of consumption of antibiotics in the community, there is a global problem of resistance. This means that geographic correlations of resistances are consistently found between different antibiotics in one species and in different species, due to the selection of coresistance and coselection of resistance by antibiotic pressure. Resistance has traditionally implied the development of new antibiotics for which basic efficacy and safety data are required prior to licensing. However, during the clinical development of a new compound, apart from collecting data on safety and tolerance, there is a need to explore the adequacy of pharmacodynamic parameters in predicting eradication (bacteriologic response) and subsequent clinical efficacy to establish breakpoints. Since evolution of bacteria towards resistance is a dynamic process, several issues should also be addressed after the introduction of a new antibiotic to the market. These issues will establish differences between the new compound and older antibiotics, and are mainly focused on the pharmacodynamic data needed to predict selection of resistance and of resistant subpopulations in multibacterial niches (simulating the nasopharynx as the specific site for emergence of resistance in respiratory tract isolates), followed in the postmarketing phase by 
specific surveillance on resistance to core antibiotics to detect emerging resistances and any link with antibiotic consumption in the community. ${ }^{88}$ This will define the role of the new antibiotic, not only from the perspective of its therapeutic potential, but also from the ecologic perspective, ie, countering resistances to core antibiotics in the community. The introduction of new antibiotics with documented adequate PK/PD and ecologic potentials might impact antibiotic policies, ie, decreased use of antibiotics with high resistance selection potential.

The documented information on cefditoren gathered from published studies in recent years, including those showing its ecologic potential in multibacterial niches, ${ }^{11,89}$ is an example of the role for an emerging oral antibacterial facing the current antibiotic resistances in community-acquired respiratory tract infections.

\section{Disclosures}

LA and MJG have received travel grants from Tedec-Meiji Farma SA, Madrid, Spain for Congress presentation of results of research carried out at the Microbiology Department, School of Medicine, University. Complutense, Madrid, Spain.

\section{References}

1. Brook I, Gober AE. Recovery of potential pathogens and interfering bacteria in the nasopharynx of otitis media-prone children and their smoking and nonsmoking parents. Arch Otolaryngol Head Neck Surg. 2005;131:509-512.

2. Sá-Leão R, Nunes S, Brito-Avô A, et al. High rates of transmission of and colonization by Streptococcus pneumoniae and Haemophilus influenzae within a day care center revealed in a longitudinal study. J Clin Microbiol. 2008;46:225-234.

3. Hammitt LL, Bruden DL, Butler JC, et al. Indirect effect of conjugate vaccine on adult carriage of Streptococcus pneumoniae: An explanation of trends in invasive pneumococcal disease. J Infect Dis. 2006;193:1487-1494.

4. Murthy TF. Haemophilus influenzae. In: Mandell GL, Bennett JE, Dolin R, editors. Principles and Practice of Infectious Diseases. 6th ed. Philadelphia, PA: Elsevier Churchill Livingstone; 2005.

5. Smith-Vaughan HC, Leach AJ, Shelby-James TM, Kemp K, Kemp DJ, Mathews JD. Carriage of multiple ribotypes of non-encapsulated Haemophilus influenzae in aboriginal infants with otitis media. Epidemiol Infect. 1996;116:177-183.

6. Musher DM. Streptococcus pneumoniae. In: Mandell GL, Bennett JE, Dolin R, editors. Principles and Practice of Infectious Diseases. 6th ed. Philadelphia, PA: Elsevier Churchill Livingstone; 2005.

7. Sá-Leão R, Tomasz A, Santos Sanches I, de Lencastre H. Pilot study of the genetic diversity of the pneumococcal nasopharyngeal flora among children attending day care centers. J Clin Microbiol. 2002;40:3577-3585.

8. Melegaro A, Choi Y, Pebody R, Gay N. Pneumococcal carriage in United Kingdom families: Estimating serotype-specific transmission parameters from longitudinal data. Am J Epidemiol. 2007;166:228-235.

9. Ekdahl K, Ahlinder I, Hansson HB, et al. Duration of nasopharyngeal carriage of penicillin-resistant Streptococcus pneumoniae: Experiences from the South Swedish Pneumococcal Intervention Project. Clin Infect Dis. 1997;25:1113-1117.
10. Bisno AL, Stevens DL. Streptococcus pyogenes. In: Mandell GL, Bennett JE, Dolin R, editors. Principles and Practice of Infectious Diseases. 6th ed. Philadelphia, PA: Elsevier Churchill Livingstone; 2005.

11. Cafini F, Aguilar L, Sevillano D, et al. Decrease in bacterial load versus resistance selection of pneumococcal subpopulations by $\beta$-lactam physiological concentrations over time: An in vitro pharmacodynamic simulation. Microbial Drug Res. 2008;14:13-21.

12. Sevillano D, Aguilar L, Alou L, et al. Effects of antimicrobials on the competitive growth of Streptococcus pneumoniae: A pharmacodynamic in vitro model approach to selection of resistant populations. J Antimicrob Chemother. 2006;58:794-801.

13. Baquero F. Modularization and evolvability in antibiotic resistance. In: Baquero F, Nombela C, Cassell GH, Gutierrez-Fuentes JA, editors. Evolutionary Biology of Bacterial and Fungal Pathogens. Washington, DC: ASM Press; 2008.

14. Courvalin P. Antimicrobial drug resistance: Prediction is very difficult, especially about the future. Emerg Infect Dis. 2005;11:1503-1506.

15. Smith DL, Laxminarayan R. Human interventions on the evolution of host-bacterium interactions. In: Baquero F, Nombela C, Cassell GH, Gutierrez-Fuentes JA, editors. Evolutionary Biology of Bacterial and Fungal Pathogens. Washington, DC: ASM Press; 2008.

16. Knudsen JD, Odenholt I, Erlendsdottir H, et al. Selection of resistant Streptococcus pneumoniae during penicillin treatment in vitro and in three animal models. Antimicrob Agents Chemother. 2003;47:2499-2506.

17. Campos J, Aracil B, Garcia-Cobos S, Oteo J. Evolution of Haemophilus influenzae and Haemophilus infections. In: Baquero F, Nombela C, Cassell GH, Gutierrez-Fuentes JA, editors. Evolutionary Biology of Bacterial and Fungal Pathogens. Washington, DC: ASM Press; 2008.

18. Takahata S, Kato Y, Sanbongi Y, Maebashi K, Ida T. Comparison of the efficacy of oral (beta)-lactams in selection of Haemophilus influenzae transformants with mutated fts I genes. Antimicrob Agents Chemother. 2008;52:1880-1883.

19. Jönsson M, Swedberg G. Macrolide resistance can be transferred by conjugation from viridans streptococci to Streptococcus pyogenes. Int J Antimicrob Agents. 2006;28:101-103.

20. Alou L, Gimenez MJ, Sevillano D, et al. A pharmacodynamic approach to antimicrobial activity in serum and epithelial lining fluid against in vivo-selected Streptococcus pneumoniae mutants and association with clinical failure in pneumonia. J Antimicrob Chemother. 2006;58:349-358.

21. Garcia-Rey C, Fenoll A, Aguilar L, Casal J. Effect of social and climatological factors on antimicrobial use and Streptococcus pneumoniae resistance in different provinces in Spain. J Antimicrob Chemother. 2004;54:465-471.

22. Llor C. Considerations for antibiotic prescription in primary care. Med Clin Monogr (Barc). 2004;5(3):52-57.

23. Mogyoros M. Challenges of managed care organisations in treating respiratory tract infections in an age of antibiotic resistance. Am J Manag Care. 2001;7(Suppl 6):163-169.

24. Huovinen P, Cars O. Control of antimicrobial resistance: Time for action. The essentials of control are already well known. BMJ. 1998;317:613-614.

25. Granizo JJ, Aguilar L, Casal J, García-Rey C, Dal-Ré R, Baquero F. Streptococcus pneumoniae resistance to erythromycin and penicillin in relation to macrolide and beta-lactam consumption in Spain (1979-1997). J Antimicrob Chemother. 2000;46:767-773.

26. García-Rey C, Aguilar L, Baquero F, Casal J, Dal-Ré R. Importance of local variations in antibiotic consumption and geographical differences of erythromycin and penicillin resistance in Streptococcus pneumoniae. J Clin Microbiol. 2002;40:159-164.

27. Gómez J, Ruiz-Gómez J, Hernández-Cardona JL, Nuñez ML, Canteras M, Valdés M. Antibiotic resistance patterns of Streptococcus pneumoniae, Haemophilus influenzae and Moraxella catarrhalis: A prospective study in Murcia, Spain, 1983-1992. Chemotherapy. 1994;40:299-303. 
28. Nissinen A, Gronroos P, Huovinen P, et al. Development of betalactamase-mediated resistance to penicillin in middle-ear isolates of Moraxella catarrhalis in Finnish children, 1978-1993. Clin Infect Dis. 1995;21:1193-1196.

29. Granizo JJ, Aguilar L, Casal J, Dal-Ré R, Baquero F. Streptococcus pyogenes resistance to erythromycin in relation to macrolide consumption in Spain (1986-1997). J Antimicrob Chemother. 2000;46:959-964.

30. García-Rey C, Aguilar L, Baquero F, Casal J, Martín JE. Pharmacoepidemiological analysis of provincial differences between consumption of macrolides and rates of erythromycin resistance among Streptococcus pyogenes isolates in Spain. J Clin Microbiol. 2002;40:2959-2963.

31. Pérez-Trallero E, García-de-la-Fuente C, García-Rey C, et al. Geographical and ecological analysis of resistance, coresistance, and coupled resistance to antimicrobials in respiratory pathogenic bacteria in Spain. Antimicrob Agents Chemother. 2005;49:1965-1972.

32. Gómez-Lus R, Granizo JJ, Aguilar L, Bouza E, Gutierrez A, García-de-Lomas J. Is there an ecological relationship between rates of antibiotic resistance of species of the genus Streptococcus? The Spanish Surveillance Group for Respiratory Pathogens. J Clin Microbiol. 1999;37:3384-3386.

33. Fenoll A, Granizo JJ, Aguilar L, et al. Temporal trends of invasive Streptococcus pneumoniae serotypes and antimicrobial resistance patterns in Spain from 1979 to 2007. J Clin Microbiol. 2009;47:1012-1020.

34. Fenoll A, Aguilar L, Granizo JJ, et al. Has the licensing of respiratory quinolones for adults and the 7-valent pneumococcal conjugate vaccine (PCV-7) for children had herd effects with respect to antimicrobial non-susceptibility in invasive Streptococcus pneumoniae? JAntimicrob Chemother. 2008;62:1430-1433.

35. Whitney CG, Farley MM, Hadler J, et al. Decline in invasive pneumococcal disease after the introduction of protein-polysaccharide conjugate vaccine. $N$ Engl J Med. 2003;348:1737-1746.

36. Pérez-Trallero E, Fernández-Mazarrasa C, García-Rey C, et al. Antimicrobial susceptibilities of 1,684 Streptococcus pneumoniae and 2,039 Streptococcus pyogenes isolates and their ecological relationships: Results of a 1-year (1998-1999) multicenter surveillance study in Spain. Antimicrob Agents Chemother. 2001;45:3334-3340.

37. Cantón R, Unal S, Farrell DJ. Antibacterial resistance patterns in Streptococcus pneumoniae isolated from elderly patients: PROTEKT years 1-5 (1999-2004). Int J Antimicrob Agents. 2007;30:546-550.

38. Pérez-Trallero E, Marimón JM, González A, García-Rey C, Aguilar L. Genetic relatedness of recently collected Spanish respiratory tract Streptococcus pneumoniae isolates with reduced susceptibility to amoxicillin. Antimicrob Agents Chemother. 2003;47:3637-3639.

39. Pérez-Trallero E, Marimón JM, Ercibengoa M, Giménez MJ, Coronel P, Aguilar L. Antimicrobial susceptibilities of amoxycillin-non-susceptible and susceptible isolates among penicillin-non-susceptible Streptococcus pneumoniae. Clin Microbiol Infect. 2007;13:937-940.

40. Baquero F, García-Rodríguez JA, García de Lomas J, Aguilar L. Antimicrobial resistance of 1,113 Streptococcus pneumoniae isolates from patients with respiratory tract infections in Spain: Results of a 1-year (1996-1997) multicenter surveillance study. The Spanish Surveillance Group for Respiratory Pathogens. Antimicrob Agents Chemother. 1999;43:357-359.

41. Jenkins SG, Farrell DJ. Increase in pneumococcus macrolide resistance, United States. Emerg Infect Dis. 2009;15:1260-1264.

42. Prieto J, Calvo A, Gómez-Lus ML. Antimicrobial resistance: A class effect? J Antimicrob Chemother. 2002;50 Suppl S2:7-12.

43. Albertí S, Cortés G, García-Rey C, et al. Streptococcus pyogenes pharyngeal isolates with reduced susceptibility to ciprofloxacin in Spain: Mechanisms of resistance and clonal diversity. Antimicrob Agents Chemother. 2005;49:418-420.

44. Alonso R, Mateo E, Galimand M, Garaizar J, Courvalin P, Cisterna R. Clonal spread of pediatric isolates of ciprofloxacin-resistant, emm type 6 Streptococcus pyogenes. J Clin Microbiol. 2005;43:2492-2493.

45. Carapetis JR, Steer AC, Mulholland EK, Weber M. The global burden of group A streptococcal diseases. Lancet Infect Dis. 2005;5:685-694.
46. Malhotra-Kumar S, Van Heirstraeten L, Lammens C, Chapelle S, Goossens H. Emergence of high-level fluoroquinolone resistance in emm6 Streptococcus pyogenes and in vitro resistance selection with ciprofloxacin, levofloxacin and moxifloxacin. JAntimicrob Chemother. 2009;63:886-894.

47. Montes M, Tamayo E, Orden B, Larruskain J, Perez-Trallero E. Prevalence and clonal characterization of Streptococcus pyogenes clinical isolates with reduced fluoroquinolone susceptibility in Spain. Antimicrob Agents Chemother. 2010;54:93-97.

48. García-Rodríguez JA, Baquero F, García de Lomas J, Aguilar L. Antimicrobial susceptibility of 1,422 Haemophilus influenzae isolates from respiratory tract infections in Spain. Results of a 1-year (1996-97) multicenter surveillance study. Spanish Surveillance Group for Respiratory Pathogens. Infection. 1999;27:265-267.

49. Marco F, García-de-Lomas J, García-Rey C, et al. Antimicrobial susceptibilities of 1,730 Haemophilus influenzae respiratory tract isolates in Spain in 1998-1999. Antimicrob Agents Chemother. 2001;45: 3226-3228.

50. Matic V, Bozdogan B, Jacobs MR, Ubukata K, Appelbaum PC. Contribution of beta-lactamase and PBP amino acid substitutions to amoxicillin/clavulanate resistance in beta-lactamase-positive, amoxicillin/ clavulanate-resistant Haemophilus influenzae. JAntimicrob Chemother. 2003;52:1018-1021.

51. Clinical and Laboratory Standards Institute. Performance standards for antimicrobial susceptibility testing; nineteenth informational supplement. CLSI document M100-S19. Wayne, PA: Clinical and Laboratory Standards Institute; 2009.

52. Jansen WT, Verel A, Beitsma M, Verhoef J, Milatovic D. Longitudinal European surveillance study of antibiotic resistance of Haemophilus influenzae. J Antimicrob Chemother. 2006;58:873-877.

53. García-Cobos S, Campos J, Lázaro E, et al. Ampicillin-resistant non-beta-lactamase-producing Haemophilus influenzae in Spain: Recent emergence of clonal isolates with increased resistance to cefotaxime and cefixime. Antimicrob Agents Chemother. 2007;51: 2564-2573.

54. MacGowan AP. Elements of design: The knowledge on which we build. Clin Microbiol Infect. 2004;10 Suppl 2:6-11.

55. Drusano GL. Antimicrobial pharmacodynamics: Critical interactions of 'bug and drug'. Nat Rev Microbiol. 2004;2:289-300.

56. Turnidge J, Paterson DL. Setting and revising antibacterial susceptibility breakpoints. Clin Microbiol Rev. 2007;20:391-408.

57. Wellington K, Curran MP. Cefditoren pivoxil: A review of its use in the treatment of bacterial infections. Drugs. 2004;64: 2597-2618.

58. Fenoll A, Giménez MJ, Robledo O, et al. Influence of penicillin/ amoxicillin non-susceptibility on the activity of third-generation cephalosporins against Streptococcus pneumoniae. Eur J Clin Microbiol Infect Dis. 2008;27:75-80.

59. Fenoll A, Giménez MJ, Robledo O, et al. Activity of cefditoren against clinical isolates of Streptococcus pneumoniae showing nonsusceptibility to penicillins, cephalosporins, macrolides, ketolides or quinolones. Int J Antimicrob Agents. 2007;29:224-226.

60. Pérez-Trallero E, Marimón JM, Ercibengoa M, Giménez MJ, Coronel P, Aguilar L. Antimicrobial susceptibilities of amoxycillin-non-susceptible and susceptible isolates among penicillin-non-susceptible Streptococcus pneumoniae. Clin Microbiol Infect. 2007;13:937-940.

61. García-de-Lomas J, Lerma M, Cebrián L, et al. Influence of Haemophilus influenzae beta-lactamase production and/or fts I gene mutations on in vitro activity of and susceptibility rates to aminopenicillins and second- and third-generation cephalosporins. Int J Antimicrob Agents. 2007;30:190-192.

62. Sevillano D, Giménez MJ, Cercenado E, et al. Genotypic versus phenotypic characterization, with respect to beta-lactam susceptibility, of Haemophilus influenzae isolates exhibiting decreased susceptibility to beta-lactam resistance markers. Antimicrob Agents Chemother. 2009;53:267-270 
63. Gracia M, Díaz C, Coronel P, et al. Antimicrobial susceptibility of Haemophilus influenzae and Moraxella catarrhalis isolates in eight Central, East and Baltic European countries in 2005-06: Results of the Cefditoren Surveillance Study. J Antimicrob Chemother. 2008;61:1180-1181.

64. Sádaba B, Azanza JR, Quetglas EG, et al. Pharmacokinetic/pharmacodynamic serum and urine profile of cefditoren following single-dose and multiple twice- and thrice-daily regimens in healthy volunteers: A phase I study. Rev Esp Quimioter. 2007;20:51-60.

65. Moellering RC, Eliopoulos GM. Principles of anti-infective therapy. In: Mandell GL, Bennett JE, Dolin R, editors. Principles and Practice of Infectious Diseases. 6th ed. Philadelphia, PA: Elsevier Churchill Livingstone; 2005.

66. Sevillano D, Aguilar L, Alou L, et al. High protein binding and cidal activity against penicillin-resistant $S$. pneumoniae: A cefditoren in vitro pharmacodynamic simulation. PLoS One. 2008;3(7):e2717.

67. Granizo JJ, Sádaba B, Honorato J, et al. Monte Carlo simulation describing the pharmacodynamic profile of cefditoren in plasma from healthy volunteers. Int J Antimicrob Agents. 2008;31:396-398.

68. Craig WA. Pharmacokinetic/pharmacodynamic parameters: Rationale for antibacterial dosing of mice and men. Clin Infect Dis. 1998;26:1-10.

69. Heffelfinger JD, Dowell SF, Jorgensen JH, et al. Management of community-acquired pneumonia in the era of pneumococcal resistance: A report from the Drug-Resistant Streptococcus pneumoniae Therapeutic Working Group. Arch Intern Med. 2000;160:1399-1408.

70. Lodise TP, Kinzig-Schippers M, Drusano GL, et al. Use of population pharmacokinetic modeling and Monte Carlo simulation to describe the pharmacodynamic profile of cefditoren in plasma and epithelial lining fluid. Antimicrob Agents Chemother. 2008;52:1945-1951.

71. US Food and Drug Administration. www.fda.gov

72. Karlowsky JA, Jones ME, Draghi DC, Critchley IA, Thornsberry C, Sahm DF. In vitro susceptibility of recent clinical isolates of pneumococci to the investigational cephalosporin cefditoren. Diagn Microbiol Infect Dis. 2002;42:59-64.

73. Johnson DM, Biedenbach DJ, Beach ML, Pfaller MA, Jones RN. Antimicrobial activity and in vitro susceptibility test development for cefditoren against Haemophilus influenzae, Moraxella catarrhalis, and Streptococcus species. Diagn Microbiol Infect Dis. 2000;37:99-105.

74. Jones RN, Biedenbach DJ, Croco MA, Barrett MS. In vitro evaluation of a novel orally administered cephalosporin (Cefditoren) tested against 1249 recent clinical isolates of Haemophilus influenzae, Moraxella catarrhalis, and Streptococcus pneumoniae. Diagn Microbiol Infect Dis. 1998;31:573-578.

75. Agencia Española de Medicamentos y Productos Sanitarios Available at: https://sinaem4.agemed.es/consaem/fichasTecnicas. do?metodo=detalleForm. Accessed on Apr 29, 2010.

76. Giménez MJ, Gómez-Lus ML, Valdés L, Aguilar L. The role of third-generation oral cephalosporin cefditoren pivoxil in the treatment of community-acquired infection in adults. Rev Esp Quimioter. 2005;18:210-216.

77. Granizo JJ, Giménez MJ, Barberán J, Coronel P, Gimeno M, Aguilar L. Efficacy of cefditoren in the treatment of upper respiratory tract infections: A pooled analysis of six clinical trials. Rev Esp Quimioter. 2008;21:14-21.

Infection and Drug Resistance

\section{Publish your work in this journal}

Infection and Drug Resistance is an international, peer-reviewed openaccess journal that focuses on the optimal treatment of infection (bacterial, fungal and viral) and the development and institution of preventive strategies to minimize the development and spread of resistance. The journal is specifically concerned with the epidemiology of antibiotic
78. Kaplan EL, Tucker RM, Poling TL, Marsh D, Chow C. Managing group A streptococcal infection. A multicenter comparison of cefditoren pivoxil and penicillin VK. J Resp Dis. 2001;22(8 Suppl):S60-S64.

79. Gooch W, Marsh D, Slickler T, Hunt B. Cefditoren is safe and effective treatment for streptococcal pharyngitis. In: Abstracts of the Fortieth Interscience Conference on Antimicrobial Agents and Chemotherapy, Toronto 2000. Abstract 837. American Society for Microbiology. Washington, USA

80. Chow J, Russell M, Volk S, Chow C. Efficacy of cefditoren pivoxil (CDTR) versus amoxicillin/clavulanate (AMX/CLV) in acute maxillary sinusitis (AMS). In: Abstracts of the Fortieth Interscience Conference on Antimicrobial Agents and Chemotherapy, Toronto 2000, Abstract 835. American Society for Microbiology, Washington, USA.

81. Granizo JJ, Giménez MJ, Barberán J, Coronel P, Gimeno M, Aguilar L. The efficacy of cefditoren pivoxil in the treatment of lower respiratory tract infections, with a focus on the per-pathogen bacteriologic response in infections caused by Streptococcus pneumoniae and Haemophilus influenzae: A pooled analysis of seven clinical trials. Clin Ther. 2006;28:2061-2069.

82. van Zyl L, le Roux JG, LaFata JA, et al. Cefditoren pivoxil versus cefpodoxime proxetil for community acquired pneumonia: Results of a multicenter, prospective, randomized, double-blind study. Clin Ther. 2002;24:1840-1853.

83. Fogarty CM, Cyganowski M, Palo WA, Hom RC, Craig WA. A comparison of cefditoren pivoxil and amoxicillin/clavulanate in the treatment of community-acquired pneumonia: A multicenter, prospective, randomized, investigator-blinded, parallel group study. Clin Ther. 2002;24:1854-1870.

84. Alvarez-Sala JL, Kardos P, Martínez-Beltrán J, Coronel P, Aguilar L. Clinical and bacteriological efficacy in treatment of acute exacerbations of chronic bronchitis with cefditoren-pivoxil versus cefuroxime-axetil. Antimicrob Agents Chemother. 2006;50:1762-1767.

85. Ramirez JA, Tucker RM, Bettis RB, et al. Treating acute exacerbations of chronic bronchitis. J Resp Dis. 2001;22(Suppl 8):S75-S80.

86. Henry DC, Poling TL, Bettis RB, et al. A double-blind, randomized study of cefditoren vs cefuroxime for AECB. J Resp Dis. 2001;22(Suppl 8): S69-S74.

87. Granizo JJ, Aguilar L, Gimenez MJ, Coronel P, Gimeno M, Prieto J. Safety profile of cefditoren: A pooled analysis of data from clinical trials in community-acquired respiratory tract infections. Rev Esp Quimioter. 2009;22:57-61.

88. Aguilar L, Giménez MJ. Gaps in antibiotic development; the postmarketing gaps. Rev Med Microbiol. 2008;19:1-7.

89. Sevillano D, Aguilar L, Alou L, et al. Beta-lactam effects on mixed cultures of common respiratory isolates as an approach to treatment effects on nasopharyngeal bacterial population dynamics. PLoS One 2008;3:e3846.

\section{Dovepress}

resistance and the mechanisms of resistance development and diffusion in both hospitals and the community. The manuscript management system is completely online and includes a very quick and fair peerreview system, which is all easy to use. Visit http://www.dovepress.com/ testimonials.php to read real quotes from published authors. 